\title{
A Gemini/GMOS study of the bright elliptical galaxy NGC 3613 and its globular cluster system
}

\author{
Bruno J. De Bórtoli, ${ }^{1,2 \star}$ Lilia P. Bassino, ${ }^{1,2}$ Juan P. Caso ${ }^{1,2}$ and Ana I. Ennis ${ }^{1,2}$ \\ ${ }^{1}$ Facultad de Ciencias Astronómicas y Geofísicas de la Universidad Nacional de La Plata, and Instituto de Astrofísica de La Plata (CCT \\ La Plata âĂS-CONICET, UNLP), Paseo del Bosque S/N, B1900FWA La Plata, Argentina \\ ${ }^{2}$ Consejo Nacional de Investigaciones Científicas y Técnicas, Godoy Cruz 2290, C1425FQB, Ciudad Autónoma de Buenos Aires, Argentina
}

Accepted XXX. Received YYY; in original form ZZZ

\begin{abstract}
We present the first photometric study of the globular cluster system (GCS) of the E galaxy NGC $3613\left(M_{\mathrm{V}}=-21.5, \mathrm{~d} \sim 30.1 \mathrm{Mpc}\right)$, as well as the surface photometry of the host galaxy, based on Gemini/GMOS images. Being considered the central galaxy of a group, NGC 3613 inhabits a low-density environment although its intrinsic brightness is similar to the expected one for galaxies in the centre of clusters. The following characteristics are obtained for this GCS. The colour distribution is bimodal, with metal-poor globular clusters (GCs) getting slightly bluer with increasing radius. The radial and azimuthal projected distributions show that metal-rich GCs are more concentrated towards the host galaxy and trace its light distribution very precisely, while metal-poor GCs present a more extended and uniform distribution. The GC luminosity function helps validate the adopted distance. The estimated total GC population of $N_{\text {tot }}=2075 \pm 130$ leads to a specific frequency $S_{N}=5.2 \pm 0.7$, a value within the expected range for GCSs with host galaxies of similar luminosity. The surface photometry of NGC 3613 reveals a three-component profile and a noticeable substructure. Finally, a small sample of ultra-compact dwarf (UCD) candidates are identified in the surroundings of the host galaxy.
\end{abstract}

Key words: galaxies: clusters: individual: NGC 3613 - galaxies: elliptical and lenticular, cD - galaxies: evolution

\section{INTRODUCTION}

The ages of globular clusters (GCs) usually establish them among the oldest objects in the Universe (e.g. Hansen et al. 2013; Tonini 2013), so they provide important clues about the first phases of galaxy formation. From the observational point of view, GCs present several advantages like being so compact and intrinsically bright that can be observed farther away than one hundred Mpc (Harris et al. 2014, 2016; Alamo-Martínez et al. 2013). Moreover, globular cluster systems (GCSs) of early-type massive galaxies contain thousands of GCs, probably as a consequence of a history of numerous mergers (e.g. Bassino et al. 2008; Durrell et al. 2014; Oldham \& Auger 2016; Caso et al. 2017).

It is often assumed that GCs formed at high redshift, in gas-rich discs and within a high-pressure environment (Kruijssen 2015). Recent numerical simulations, like the EMOSAICS Project (Pfeffer et al. 2018; Kruijssen et al. 2019),

\footnotetext{
^ E-mails: brudebo.444@gmail.com, liliaybass@gmail.com, jpceda@gmail.com, ennis.ana@gmail.com
}

have presented scenarios that describe the formation, evolution and disruption of the GCs, following their evolution together with that of the host galaxies. These scenarios imply a direct correlation between the formation of GCs and the field stars, in such a way that the properties of GCSs provide constraints to the simulations (e.g. Powalka et al. 2016) and, on the other side, a galaxy history can be described based on the study of its GCS. Such interconnections follow clearly from studies of large GC samples, like the ACS Fornax Cluster Survey (ACSFCS) (Jordán et al. 2007) or the Next Generation Virgo Cluster Survey (NGVS) (Ferrarese et al. 2012).

One of the most common characteristics of GCSs in massive early-type galaxies is the existence of two GC subpopulations, though more complex cases have been pointed out (e.g. Caso et al. 2013; Sesto et al. 2016). These GC subpopulations have been detected through different physical properties:

- bimodality in colour, which is interpreted mainly as a difference in metallicity for the bona fide old GCs, where 'blue' and 'red' subpopulations identify those with lower and 
higher metal content (i.e. metal-poor and metal-rich GCs), respectively (e.g. Usher et al. 2012; Chies-Santos et al. 2012; Forte et al. 2013).

- different projected spatial distribution with respect to the host galaxy, with red GCs being generally more concentrated towards the centre of the host galaxies and tracing their surface-brightness profiles, while blue ones present a more extended distribution (e.g. Bassino et al. 2006; Forbes et al. 2012; Durrell et al. 2014; Escudero et al. 2018).

- different kinematics, found in the radial velocity and velocity dispersion of the subpopulations. The kinematics of the red subpopulation is usually akin to that of the host galaxy stars (e.g. Schuberth et al. 2010; Pota et al. 2013; Amorisco 2019). According to the numerical simulations by Amorisco (2019), the higher dispersion of blue GCs relative to red ones may be explained by the high contribution of blue clusters to the halo population through minor mergers.

Our current target, NGC 3613, is an intrinsically bright elliptical galaxy, classified as E6 (de Vaucouleurs et al. 1991). We initially adopt a distance d 30.1 Mpc (Tully et al. 2013), based on surface brightness fluctuations, but taking into account that the distances calculated to date have a significant dispersion, as can be seen in $\mathrm{NED}^{1}$. In particular, one of the aims of this work is to provide a new estimate for this value by means of the turn-over of the globular cluster luminosity function. Then, the absolute visual magnitude of NGC 3613 $\left(M_{\mathrm{V}}=-21.5\right)$ corresponds to the range of those of bright massive galaxies located in rich clusters, although it is noticeable that it inhabits an environment of lower density .

The ATLAS ${ }^{3 D}$ project (Cappellari et al. 2011a), a survey that combines multi-wavelength data and models, includes NGC 3613 in its sample of 260 early-type galaxies. According to their kinematic analysis (Krajnović et al. 2011), our target is a 'regular rotator' (i.e. dominated by ordered rotation) and, based on an estimator of the angular momentum of the stars, it is also classified as a 'fast rotator' (Emsellem et al. 2011). The local density estimators presented by Cappellari et al. (2011b), place NGC 3613 in a low-density environment. They also state that fast rotators form a homogeneous category of systems flattened and oblate, with regular velocity fields. One of the last papers of the $\operatorname{ATLAS}^{3 D}$ project deals with the stellar populations of the early-type galaxy sample (McDermid et al. 2015), and gives values of the age and metallicity of NGC 3613, measured within the effective radius, calculated by two methods. Using single stellar population models they obtain: age = $11 \pm 2$ Gyr and $[\mathrm{Z} / \mathrm{H}]=-0.17 \pm 0.05$, and using spectral fitting to derive star formation history, they obtain mass-weighted values of age $=13 \pm 0.7 \mathrm{Gyr}$ and $[\mathrm{Z} / \mathrm{H}]=-0.13 \pm 0.01$. Under both approaches, our target turns out to be a quite old and metal-poor galaxy.

More recently, O'Sullivan et al. (2017) presented the Complete Local-Volume Groups Sample (CLoGS) that includes 53 optically-selected groups located in the nearby Universe, up to a distance of $80 \mathrm{Mpc}$. According to their selection criterion (i.e. considering the brightest early-type member of the group as the central galaxy), NGC 3613 is not only member of a group but is also the central galaxy.

1 https://ned.ipac.caltech.edu/
As far as we know, the GCS of NGC 3613 has not been studied before, which is surprising given that the host is such a bright galaxy. According to the study of Madore et al. (2004), NGC 3613 belongs to a group consisting of a dozen galaxies. Located at an angular distance of $47 \mathrm{arcmin}$ towards the north and with a radial velocity difference of $350 \mathrm{~km} \mathrm{sec}^{-1}$, there is a peculiar lenticular (also classified as shell elliptical) galaxy of similar luminosity, NGC 3610, that is considered as a prototype of a merger remnant of two disc galaxies. The latter galaxy has a very complex surfacebrightness distribution with plumes, tails and other structures as a consequence of the tidal disturbances suffered during its evolution (Schweizer \& Seitzer 1992; Bassino \& Caso 2017). Madore et al. (2004) indicate that both galaxies might belong to the same group, which may then have undergone mergers and tidal-stripping processes. Moreover, the estimated projected distance between them $(\approx 400 \mathrm{kpc}$ assuming they are both at the same distance) lends support to the idea that they may have formed in a common environment.

Thus, the current analysis of NGC 3613 and its GCS will not only allow us to characterize the system and confirm its distance, but also look for evidence of possible interactions with other group members, e.g. by detecting spatial enhancements or irregularities in the projected GC azimuthal distribution, substructure in the host surface-brightness distribution, etc.

This paper is organised as follows. The observations and data reduction are described in Sections 2 and 3, while the results are presented in Section 4. In Section 5 we analyse the surface photometry of the galaxy and present our discussion in Section 6. A summary and conclusions are given in Section 7.

\section{OBSERVATIONS}

The data were obtained with Gemini/GMOS-N during semester 2013A (programme GN2013A-Q-42, PI: J.P. Caso), in nights with photometric quality, and consist of images of the galaxies NGC 3610 and NGC 3613 in $g^{\prime}, r^{\prime}$, and $i^{\prime}$ bands. Fig. 1 shows the configuration of the observed fields. The images of NGC 3610 (one field on the galaxy plus another 'adjacent' field) have been used previously to study both the galaxy and its GCS (Bassino \& Caso 2017), while those of NGC 3613 (one field on the galaxy) are the ones used in the present study to analyse the properties of the GCS of NGC 3613. In order to estimate the contamination by Galactic stars and background galaxies, we will use half of the 'adjacent field' (the one that is further from the centre of NGC 3610). This field is close in projection and has been taken as part of the same programme. Moreover, the GCS of NGC 3610 extends up to a galactocentric radius of $\sim 4$ arcmin, so almost no GCs are expected in this half of the 'adjacent field'.

The observing $\log$ is presented in Table 1. Four longexposures were taken for each band. We also note that the $g^{\prime}$ images were obtained on two different nights.

A dithering pattern was used to cover the gaps and remove cosmic rays and bad pixels, as well as a $2 \times 2$ binning, resulting in a scale of 0.146 arcsec pixell ${ }^{-1}$.

For the data reduction, we used tasks of the GEM- 


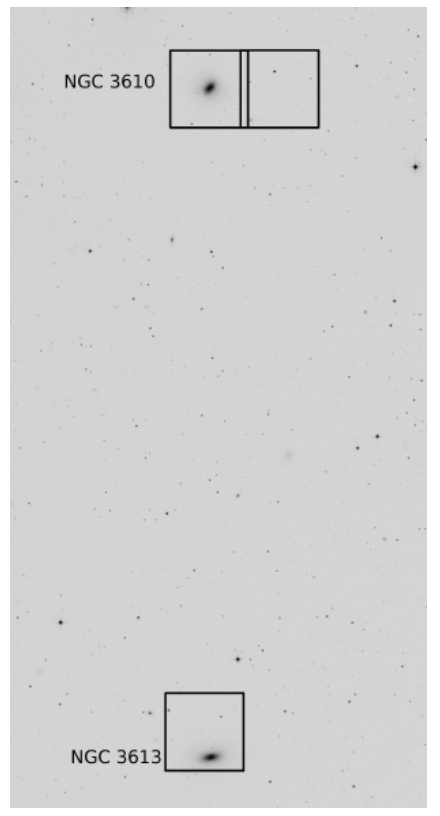

Figure 1. POSS image in $R$-band (field of view FOV: $28 \times$ 54 arcmin). North is up, east to the left. The three GMOS fields (FOV: $5.5 \times 5.5$ arcmin) observed with our programme are superimposed. The angular distance between both galaxies is approximately 47 arcmin. Half of the upper right field is used as a comparison field in the present work.

Table 1. Observation log

\begin{tabular}{lccr}
\hline Filter & $\mathrm{N}$ & Date & Exposure time \\
\hline \hline$g^{\prime}$ & 2 & $08 / 03 / 2013$ & $450 \mathrm{sec}$ \\
\hline$g^{\prime}$ & 2 & $13 / 02 / 2013$ & $450 \mathrm{sec}$ \\
\hline$r^{\prime}$ & 4 & $13 / 02 / 2013$ & $210 \mathrm{sec}$ \\
\hline$i^{\prime}$ & 4 & $13 / 02 / 2013$ & $270 \mathrm{sec}$ \\
\hline
\end{tabular}

INI package (in particular, GMOS package) and IRAF DAOPHOT.

\section{PHOTOMETRY}

\subsection{Point source selection and photometry}

In order to improve the detection of GC candidates located near the centre of the galaxy and to remove possible gradients in the surface-brightness distribution, we subtracted the light of the galaxy as much as possible using the task FMEDIAN, applying first a filter that calculates the median value in squares of $200 \times 200$ pixels, and then repeating the procedure with one of $40 \times 40$ pixels to eliminate fluctuations of lower period. With the photometry of the artificial stars (see section 3.3) we corroborated that this procedure does not modify the results obtained for point sources.

To obtain an initial catalog of point-sources present in the GMOS field, we used the software SEXTRACTOR (Bertin $\&$ Arnouts 1996). We ran the software on all $g^{\prime}, r^{\prime}$ and $i^{\prime}$ images using two filters, one (Gaussian) that is more effective at larger distances from the galaxy, and another (Mexhat) which performs a better fit in highly populated areas such as those near the centre of the galaxy, where candidates for GCs are concentrated. The program generates a catalog for both cases, Mexhat and Gaussian filters. Then, we selected those objects listed in at least one catalog for $g^{\prime}, r^{\prime}$ and $i^{\prime}$, and with a parameter CLASS_STAR greater than 0.4 to eliminate extended sources.

We performed PSF photometry with the corresponding tasks of the DAOPHOT package within IRAF. For each filter, a PSF model was obtained with about 20 isolated bright stars, well distributed over the field. The ALLSTAR task also gave us statistical parameters ( $\chi$ and sharpness). By means of these parameters, a new improved point-source catalog was obtained.

Finally, aperture corrections were estimated using the same objects as those used to obtain the respective PSFs.

\subsection{Photometric calibration}

As part of the Gemini programme, a standard star field from the list of Smith et al. (2002) was observed, and reduced in the previous study of NGC 3610 by Bassino \& Caso (2017). To obtain magnitudes in the standard system, the calibration equation for each filter is:

$m_{\text {std }}=Z P+m_{\text {inst }}-K_{M K}(X-1.0)$

where $m_{\text {std }}$ and $m_{\text {inst }}$ are the standard and instrumental magnitudes, respectively, $Z P$ is the photometric zero-point, $K_{M K}$ the mean atmospheric extinction at Mauna Kea (obtained from the Gemini Observatory Web Page ${ }^{2}$ ), and $X$ the airmass. In the present work, the same calibration equations as those obtained by Bassino \& Caso (2017) were applied.

Finally, we applied the corrections by Galactic extinction obtained from NED, which were calculated by Schlafly \& Finkbeiner (2011).

\subsection{Completeness estimation}

In order to estimate the photometric completeness for our fields, first we added to the images 250 artificial stars, uniformly distributed, covering a magnitude range $21.5 \leq i_{0}^{\prime} \leq$ 27 and the expected colour range for GCs. We repeated this procedure 40 times, achieving a sample of 10000 artificial stars in each image. Then, we performed the detection and photometry in the same way as in the original science images. The process was carried out for four ranges of galactocentric radii. In addition, it was repeated for the comparison field in order to estimate the contamination corrected by completeness. The resulting completeness curves are shown in Figs. 2 and 3.

The fitted function (Harris 2009) is:

$f\left(i_{0}^{\prime}\right)=\beta\left(1-\frac{\alpha\left(i_{0}^{\prime}-m_{0}\right)}{\sqrt{1+\alpha^{2}\left(i_{0}^{\prime}-m_{0}\right)^{2}}}\right)$

where $\alpha, \beta$ and $m_{0}$ are the free parameters.

2 http://www.gemini.edu/sciops/instruments/gmos/calibration 


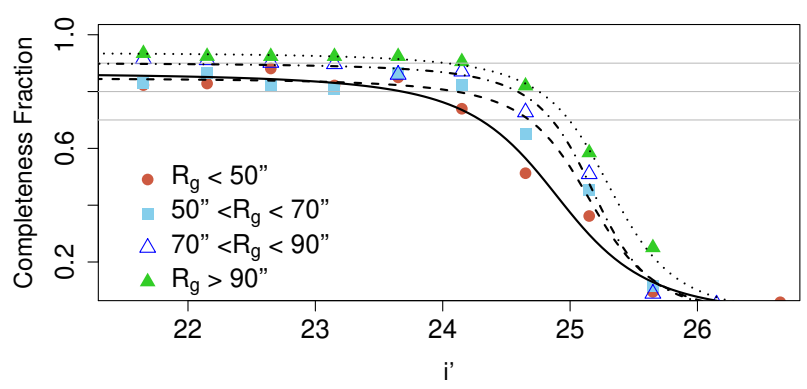

Figure 2. Completeness curves for the science field as a function of $i_{0}^{\prime}$. Different line types represent the fits for the denoted $R_{g}$ ranges. Horizontal lines indicate 70, 80 and 90 per cent completeness.

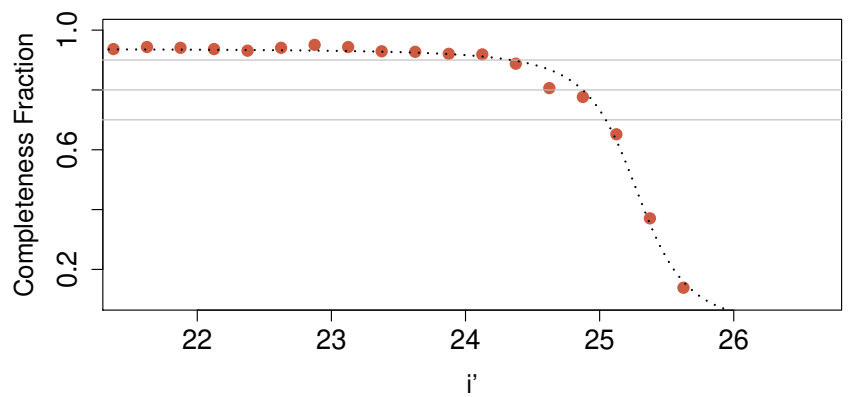

Figure 3. Completeness curve for the comparison field as a function of $i_{0}^{\prime}$. Dotted line represents the fit. Horizontal lines as in Fig. 2.

Hereafter, the limit $i_{0}^{\prime}<25$ is used to guarantee an acceptable completeness in both, the science and comparison fields.

\section{RESULTS}

\subsection{Selection of GC candidates}

The GC candidates will be selected among the point-sources, according to certain brightness and colour ranges. On the one hand, the faint magnitude limit was determined in the previous Section according to the adopted completeness for the science images (i.e. $\left.i_{0}^{\prime} \sim 25\right)$. On the other hand, the bright magnitude limit will be taken as the estimated limiting magnitude that separates Ultra Compact Dwarf (UCD) and bright $\mathrm{GC}$ candidates. Adopting as such limit the $M_{I}$ magnitude derived from Mieske et al. (2006) (i.e. $M_{I}=-12$ ), using the transformation equations given by Faifer et al. (2011) and the adopted distance for NGC 3613, we calculate the bright magnitude limit as $i_{0}^{\prime}=20.8$.

In regards to the colour range, Fig. 4 shows the colourcolour diagrams, $\left(r^{\prime}-i^{\prime}\right)_{0}$ versus $\left(g^{\prime}-i^{\prime}\right)_{0}$ and $\left(g^{\prime}-r^{\prime}\right)_{0}$ versus $\left(g^{\prime}-i^{\prime}\right)_{0}$ for the selected point-sources. The use of colour-colour diagrams to select GC candidates has been thoroughly explained by Faifer et al. (2011). This method has proved to result in a clean selection of GCs, with only a
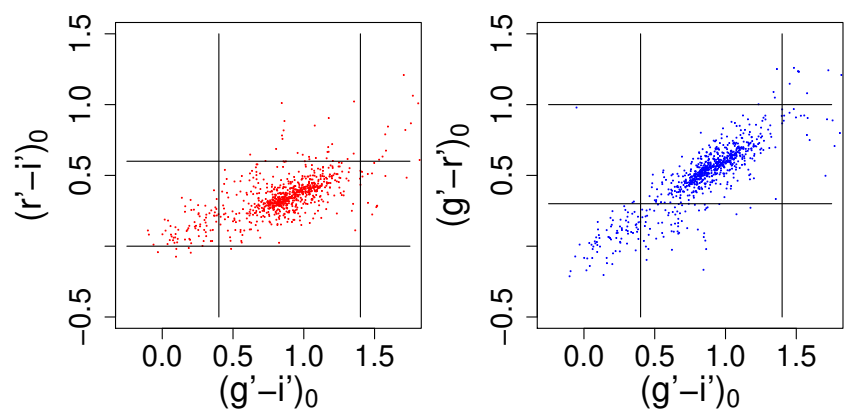

Figure 4. Colour-colour diagrams for the selected point-sources. Solid lines indicate the typical colour limits for GC candidates.
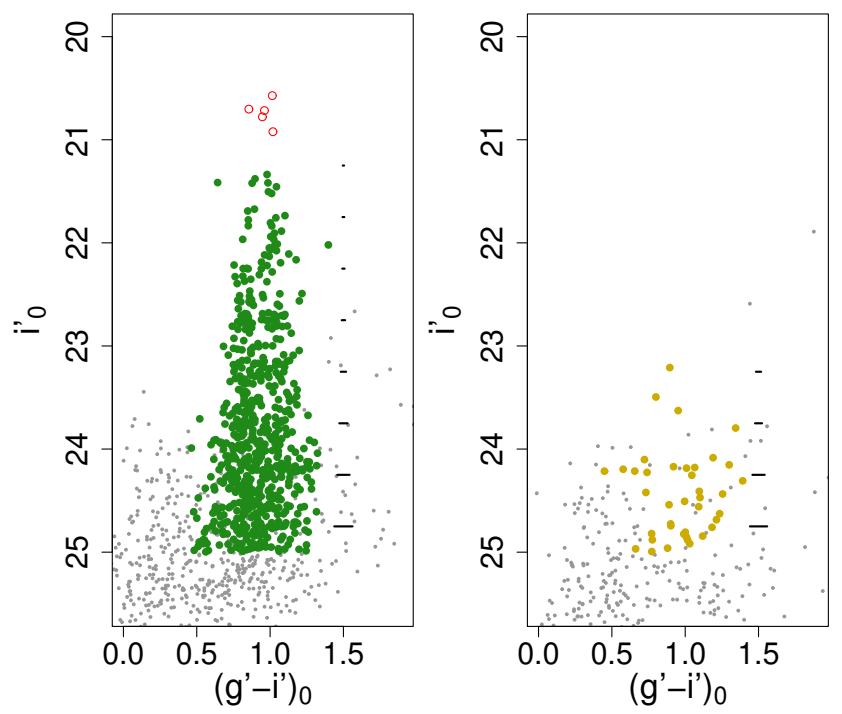

Figure 5. Colour-magnitude diagram for the science field (left panel) and the comparison field (right panel). The highlighted points represent those specific objects that fulfill the criteria adopted for selecting GC candidates. The open circles indicate UCD candidates (see Discussion). The colour errors for different magnitudes are displayed on the right side of both diagrams.

small fraction of contaminants, when spectroscopic observations to confirm membership are available (e.g. Norris et al. 2008, 2012, and references therein) Accordingly, there are well-defined sequences in these diagrams that are indicated by the solid lines. We then select as GC candidates those in the colour ranges $0.4<\left(g^{\prime}-i^{\prime}\right)_{0}<1.4,0.0<\left(r^{\prime}-i^{\prime}\right)_{0}<0.6$ and $0.3<\left(g^{\prime}-r^{\prime}\right)_{0}<1$ (Caso et al. 2015; Escudero et al. 2015 , and references therein).

Finally, Fig. 5 shows the colour-magnitude diagram $i_{0}^{\prime}$ versus $\left(g^{\prime}-i^{\prime}\right)_{0}$ for the science field (left panel) and for the comparison field (right panel). The locus of the bona-fide GC candidates appears clearly on the science field, even the two subpopulations can be distinguished at first sight. In the comparison field, contaminants that fulfill the same criteria as GC candidates are present only for $i_{0}^{\prime}>23$, with a total of 4.9 objects $/ \operatorname{arcmin}^{2}$. 


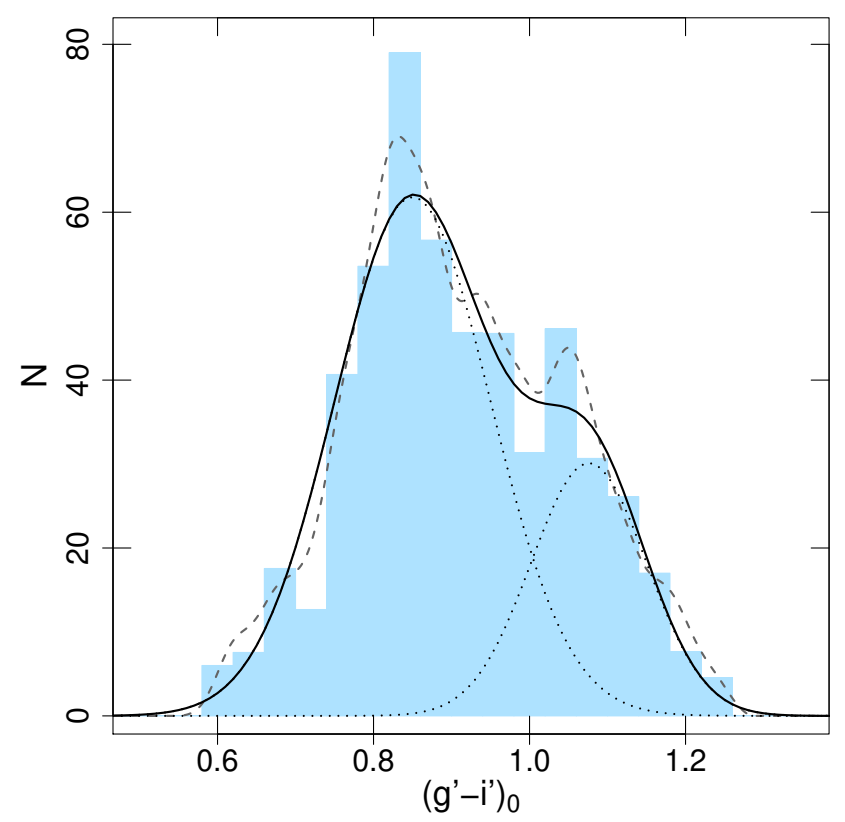

Figure 6. Colour-distribution for GC candidates. Dashed and dotted lines show a smoothed histogram (with a $0.5 \sigma$ Gaussian kernel)

and the two fitted Gaussians, respectively. Solid line shows the result of adding these Gaussians.

\subsection{Colour distribution}

Fig. 6 shows the $\left(g^{\prime}-i^{\prime}\right)_{0}$ colour distribution for all GC candidates, using a bin width of 0.04 mag. A smoothed histogram (with a $0.5 \sigma$ Gaussian kernel) is also shown with dashedlines. We note that for this analysis, the central zone of the galaxy is excluded due to saturation.

In order to analyse whether the global colour distribution can be represented as the sum of two Gaussian models, we used the Gaussian Mixture Modeling test (GMM, Muratov \& Gnedin 2010). By means of the GMM test, we fitted two Gaussians to the sample, obtaining mean value, dispersion, and fraction for each subpopulation, i.e. metal-poor ('blue') and metal-rich ('red') GC candidates. The test also gives two statistical parameters, DD and the kurtosis of the input distribution. The DD parameter is a measure of the separation between the peaks of the two Gaussians, calculated as:

$D D=\frac{\left|\mu_{1}-\mu_{2}\right|}{\left(\sigma_{1}^{2}+\sigma_{2}^{2}\right)^{1 / 2}}$

where $\mu_{1}$ and $\mu_{2}$ are the mean values and $\sigma_{1}$ and $\sigma_{2}$ the dispersions of the fitted Gaussians. A bimodal distribution is acceptable when $\mathrm{DD}>2$, while the kurtosis is very likely negative in such a case.

In order to run GMM on contamination-free samples, we proceeded as follows. The expected number of contaminants, $N_{c}$, was calculated for each region, taking into account the ratio between the areas covered by the sub-sample and the comparison field. Due to the fact that the regions in which the sample was divided present a smaller area than that corresponding to the comparison field, we proceeded to randomly select $N_{c}$ objects from the comparison field, to

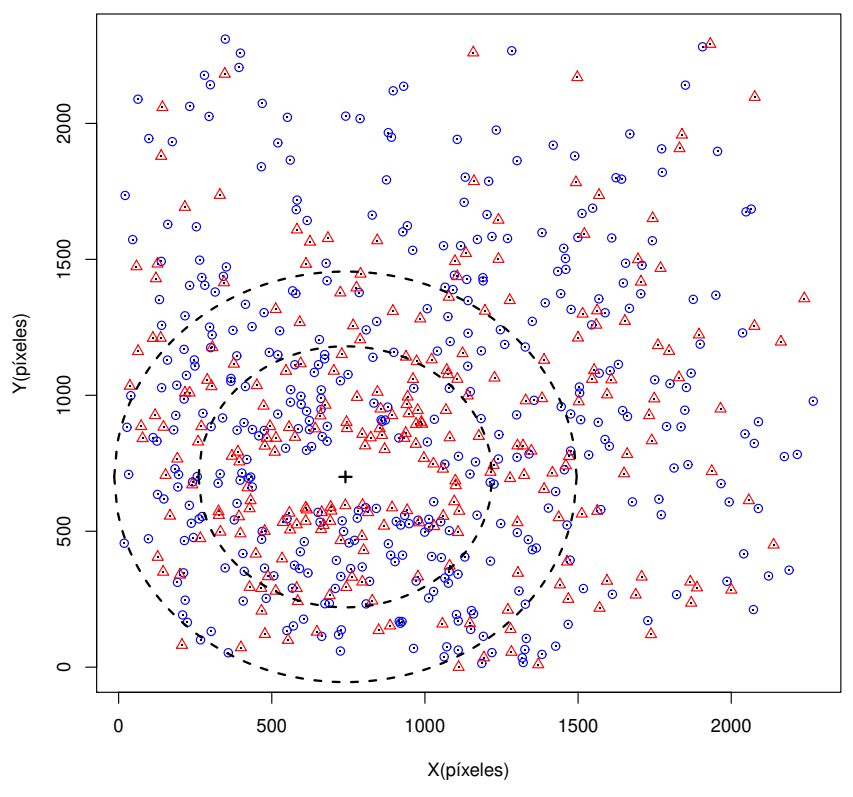

Figure 7. Projected spatial distribution of blue and red GC candidates, indicated with blue circles and red triangles, respectively. The dashed lines show the three radial ranges used to study the colour distribution. The galaxy centre is marked with a cross.

then subtract from the science sample those that present more similar colours to each of them. This random selection can introduce some statistical noise. To minimize this effect, the procedure was repeated 25 times and the results were averaged to obtain the final parameters of each fitted Gaussian.

The results are listed in Table 2, where it can be seen that for the whole sample it is acceptable to consider a bimodal distribution.

We also performed this analysis for three concentric regions. We separated them according to the following galactocentric radii $\left(R_{g}\right): 20<R_{g}<70 \operatorname{arcsec}, 70<R_{g}<110$ arcsec, and $R_{g}>110$ arcsec (see Fig. 7), using a bin width of 0.06 mag. Fig. 8 depicts the three colour distributions and Table 2 shows the corresponding results of the GMM test. According to the DD parameters and kurtosis obtained, it is also acceptable to consider bimodal distributions for the subsamples in the three concentric regions. As the $f_{\text {red }}$ parameter indicates, the blue subpopulation dominates clearly in all galactocentric ranges, unlike other bright elliptical galaxies where in the innermost region the weight of both subpopulations is similar (e.g. Caso et al. 2019).

As can be noticed from Table 2, mean $(g-i)_{0}^{\prime}$ colours of blue and red subpopulations remain approximately at similar values for the three subsamples and for the total population, except that the blue peak gets bluer with increasing radius (we will come back to this in the Discussion) and the red peak of the intermediate region is bluer than the rest, though the latter also has the largest error. Globally, these mean values mostly agree with those found in other studies of GCSs in the same photometric system, that is $\mu \approx 0.85$ and $\mu \approx 1.07$ for the blue and red peaks, respectively (e.g. Harris 2009; Forbes et al. 2011, and references therein). Moreover, the fraction of metal-rich clusters in the inner and intermediate regions is larger than in the outer- 

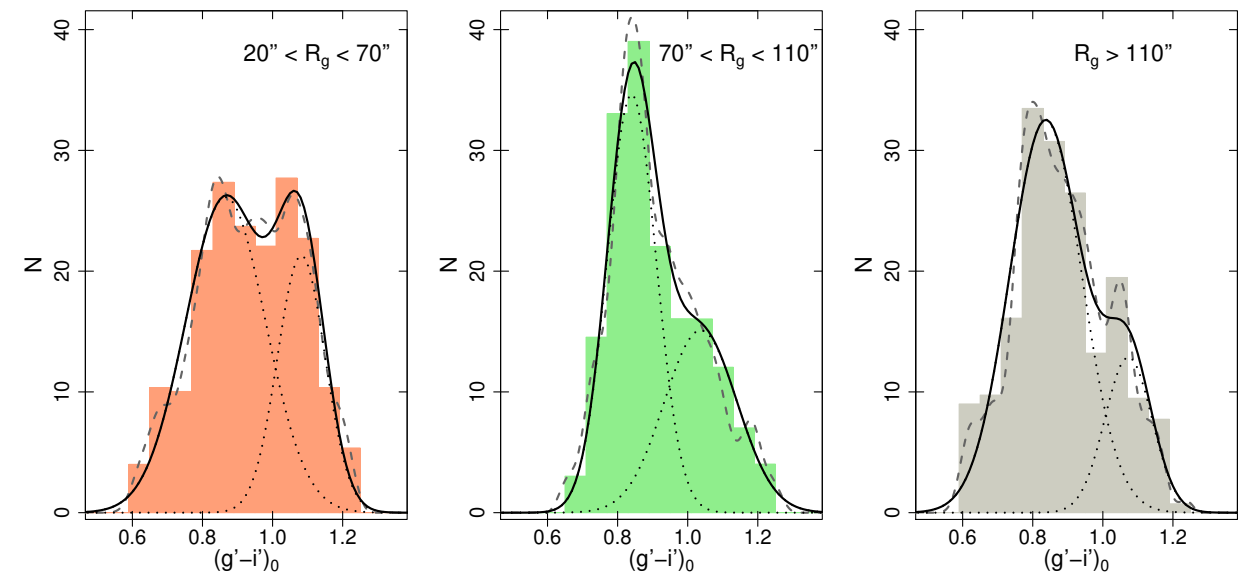

Figure 8. Colour distribution for GC candidates, for three different radial regimes. Dashed, dotted, and solid lines as in Fig. 6.

Table 2. Parameters obtained by fitting two Gaussians with the GMM test, for different radial ranges. $\mu_{1}, \sigma_{1}, \mu_{2}, \sigma_{2}$ correspond to the mean value and dispersion in $\left(g^{\prime}-i^{\prime}\right)_{0}$ for the blue and red subpopulations, respectively. DD and kurt (kurstosis) are obtained with GMM. The fraction of red GCs $f_{r} e d$, is depicted in the last column.

\begin{tabular}{cccccccc}
\hline Region & $\mu_{1}$ & $\sigma_{1}$ & $\mu_{2}$ & $\sigma_{2}$ & DD & kurt & $f_{\text {red }}$ \\
\hline \hline Total & $0.850 \pm 0.003$ & $0.100 \pm 0.002$ & $1.076 \pm 0.006$ & $0.074 \pm 0.005$ & $2.538 \pm 0.085$ & -0.573 & $0.265 \pm 0.020$ \\
\hline $20 "<R_{g}<70 ”$ & $0.866 \pm 0.006$ & $0.114 \pm 0.002$ & $1.082 \pm 0.005$ & $0.068 \pm 0.003$ & $2.294 \pm 0.058$ & -0.776 & $0.327 \pm 0.024$ \\
\hline $70 "<R_{g}<110 ”$ & $0.840 \pm 0.006$ & $0.070 \pm 0.008$ & $1.037 \pm 0.029$ & $0.104 \pm 0.010$ & $2.149 \pm 0.359$ & -0.226 & $0.393 \pm 0.097$ \\
\hline$R_{g}>110 ”$ & $0.837 \pm 0.009$ & $0.103 \pm 0.003$ & $1.073 \pm 0.019$ & $0.067 \pm 0.016$ & $2.634 \pm 0.251$ & -0.486 & $0.205 \pm 0.042$ \\
\hline
\end{tabular}

most one, which is in agreement with the idea that this red subpopulation is more concentrated towards the host galaxy and thus, closely related to its stellar component.

\subsection{Blue-tilt}

In the colour-magnitude diagram depicted in Fig. 5, it can be clearly seen that as we consider brighter blue GC candidates, they get redder. This behaviour has been generally called 'blue-tilt' and, in our case, it extends over the whole luminosity range. Also, some authors refer to it as a 'massmetallicity relation (MMR)' (e.g. Harris et al. 2006), applied to this colour-luminosity trend followed by the metal-poor GCs in many bright galaxies, but not all of them.

In order to characterize the blue-tilt, Fig. 9 shows the colour-magnitude diagram, differentiating the red and blue GC candidates by taking $(g-i)_{0}^{\prime}=0.95$ (Faifer et al. 2011) as limiting colour between both subpopulations. In addition, the large dots represent the mean colour of different adjacent subsamples in each subpopulation, each subsample with equal number of GC candidates (50 for the red candidates and 65 for the blue ones). It can be seen that in the case of the blue GC candidates, a correlation between colour and magnitude is present, as mean colours are tilted towards the red as we consider brighter GCs. By means of a linear leastsquares fit of those mean blue colours we obtained a slope of $d\left(g^{\prime}-i^{\prime}\right)_{0} / d i_{0}^{\prime}=-0.053 \pm 0.015$ (the result of a chi-square test indicates that the fit represents the distribution with a
90 per cent of confidence.). Thus, it is in agreement within uncertainties to that obtained, in the same photometric system, by Wehner et al. $(2008)\left(d\left(g^{\prime}-i^{\prime}\right)_{0} / d i_{0}^{\prime}=-0.044 \pm 0.011\right)$ for NGC 3311, the central galaxy of the Hydra cluster, and slightly larger than the one obtained by Escudero et al. $(2015)\left(d\left(g^{\prime}-i^{\prime}\right)_{0} / d i_{0}^{\prime}=-0.026 \pm 0.007\right)$ for a bright lenticular, NGC 6861 .

\subsection{Projected spatial and radial distributions}

Fig. 10 shows the projected spatial distribution of the GC candidates surrounding the galaxy NGC 3613. It is divided into blue and red GC subpopulations, according to the adopted colour limit, $(g-i)_{0}^{\prime}=0.95$. The corresponding projected density is superimposed as a smoothed distribution as well as a few contours of constant numerical density.

As already indicated by the decreasing fraction of red GCs with galactocentric distance, it is clear from Fig. 10 that the red GC subpopulation is more concentrated towards the centre of the galaxy, while the blue subpopulation is more extended and evenly distributed in an approximately circular distribution. The contours of the red GCs are elliptical, with the major axis oriented in a similar direction as the host galaxy starlight.

The projected radial distributions for all GC candidates and for both subpopulations, corrected by contamination and by completeness, are presented in Fig. 11. All the radial 


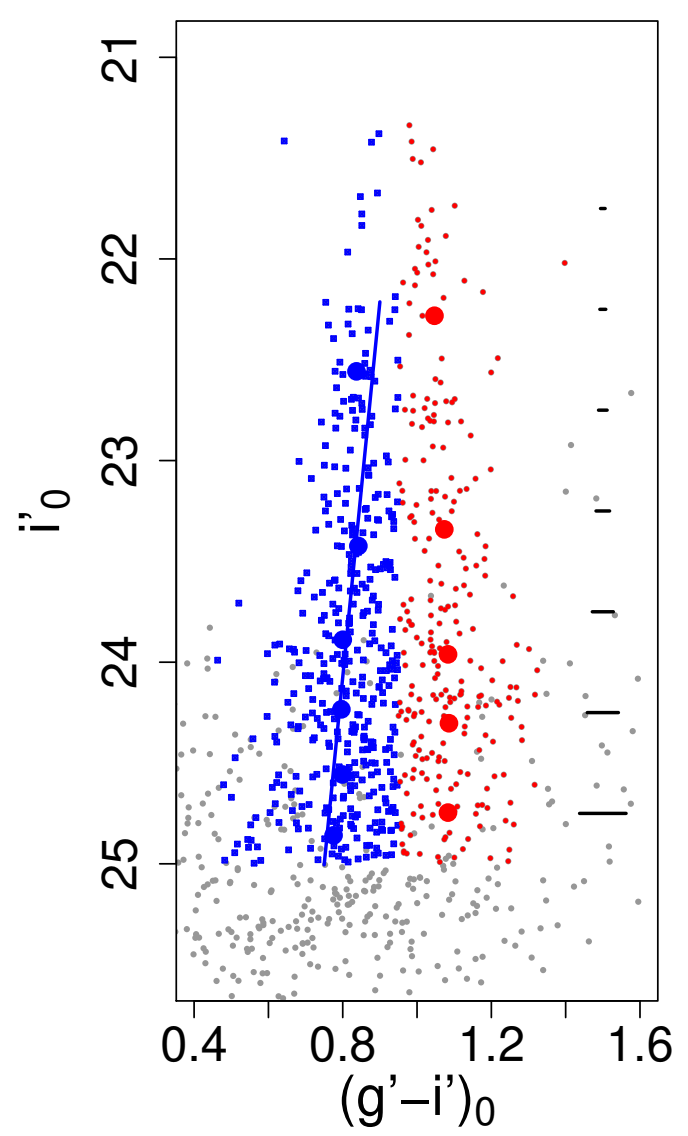

Figure 9. Colour-magnitude diagram for point sources (small dots). Metal-poor (blue) and metal-rich (red) GC candidates are shown with squares and filled circles respectively. Large dots represent colour averages of different subsamples, sorted in magnitude, with equal number of candidates.

profiles were fitted with power-laws to calculate the respective slopes. Due to saturation at the galaxy centre, the fits were performed for $\mathrm{r}>0.35 \mathrm{arcmin}$. According to the powerlaw,

$\log _{10}(N)=d+e \log _{10}(r)$

$r$ is the galactocentric radius and $\mathrm{d}$, e are the fitted coefficients. The corresponding results are depicted in Table 3. As can be seen in Fig. 11, the power-law provides good fits for the blue and red subpopulations, excluding from the fit of the latter subpopulation the furthermost point. However, the power-law fit is not as good for the case of the whole sample.

Then, a modified Hubble distribution (Binney \& Tremaine 1987) was also fitted to the whole sample profile, within the same radial range, to take into account the evident change of slope present in the profile. In previous works we have obtained good fits this way (e.g. Bassino \& Caso 2017; Caso et al. 2017). By means of the Hubble profile,

$n(r)=a\left(1+\left(\frac{r}{r_{0}}\right)^{2}\right)^{b}$

where $r$ is the galactocentric radius and $a, b, r_{0}$ are the fit-

\begin{tabular}{cccc}
\hline & All & Blue & Red \\
\hline \hline $\mathrm{d}$ & $1.70 \pm 0.03$ & $1.47 \pm 0.03$ & $1.33 \pm 0.03$ \\
\hline $\mathrm{e}$ & $-1.21 \pm 0.12$ & $-0.97 \pm 0.10$ & $-1.30 \pm 0.11$ \\
\hline
\end{tabular}

Table 3. Coefficients of the power-law fitted to the radial profiles for all, blue, and red GC candidates.

ted coefficients, we obtained the following values $a=137 \pm$ $15 \mathrm{Narcmin}^{-2}, \quad r_{0}=1.04 \pm 0.24 \operatorname{arcmin}$ and $b=-1.15 \pm 0.19$. This fit is better than the one obtained with a power-law, particularly for the innermost points where the destruction of GCs (Kruijssen et al. 2012; Kruijssen 2015) must have affected the profile. As a third option, we fitted a SÃl'rsic model (Sersic 1968) to the whole GC sample, that resulted quite similar to that of the Hubble profile and gave an effective radius $R_{\text {eff }}=1.97 \pm 0.16 \operatorname{arcmin}(17 \mathrm{kpc})$, for the total projected GC distribution (Fig. 11, upper panel). This value is slightly larger than those obtained by Usher et al. (2013) for NGC $4278(12.7 \mathrm{kpc})$, and Kartha et al. (2014) for NGC 720 and NGC 2768 (13.4 kpc and $10.5 \mathrm{kpc}$, respectively), all $\mathrm{E}$ galaxies with similar luminosity than NGC 3613.

We assume that the total extension of the GCS is reached at the radius where the background-corrected density, corresponding to the Hubble profile, is equal to 30 per cent of the background level. Such a criterion was first used by Bassino et al. (2006) in a wide-field study of the GCS of NGC 1399, based on three MOSAIC II (CTIO) images (FOV: $36 \times 36$ arcmin each). The galactocentric radius corresponding to 30 per cent of the background was the largest distance from the host galaxy where GCs and the background could be separated, being the density distribution flat further out. If we consider this limit, which has also been adopted in subsequent works (e.g. Caso et al. 2013; Bassino \& Caso 2017), the GCS of NGC 3613 exceeds the FOV of our images. Thus, we obtain an extension of $r=8.1 \mathrm{arcmin}$, that is $r=70 \mathrm{kpc}$.

\subsection{Azimuthal distribution}

Fig. 12 shows how the GC subpopulations are distributed with respect to the position angle (PA), which is measured from north to east with vertex at the galaxy centre. Such distributions were estimated considering an annulus defined by the largest possible outer radius so as the whole annulus was contained within the FOV, i.e. $48<R_{g}<102$ arcsec. It was divided into angular sections of $\approx 30^{\circ}$ and the GC number density was calculated for each bin. It can be seen that the blue GCs do not show any particular behavior, as it is basically a rather uniform distribution, except for a slight drop at $\mathrm{PA} \sim 300^{\circ}$. On the other hand, the red GCs show a sinusoidal behavior, with two clear over-densities at PA that differ approximately by $180^{\circ}$. As expected, the position of these over-densities agrees with what is obtained from the contours of constant density at the GC projected spatial distribution (see Fig. 10), defining the same direction as the ellipse major axis.

In order to fit the red GC distribution, we used the 

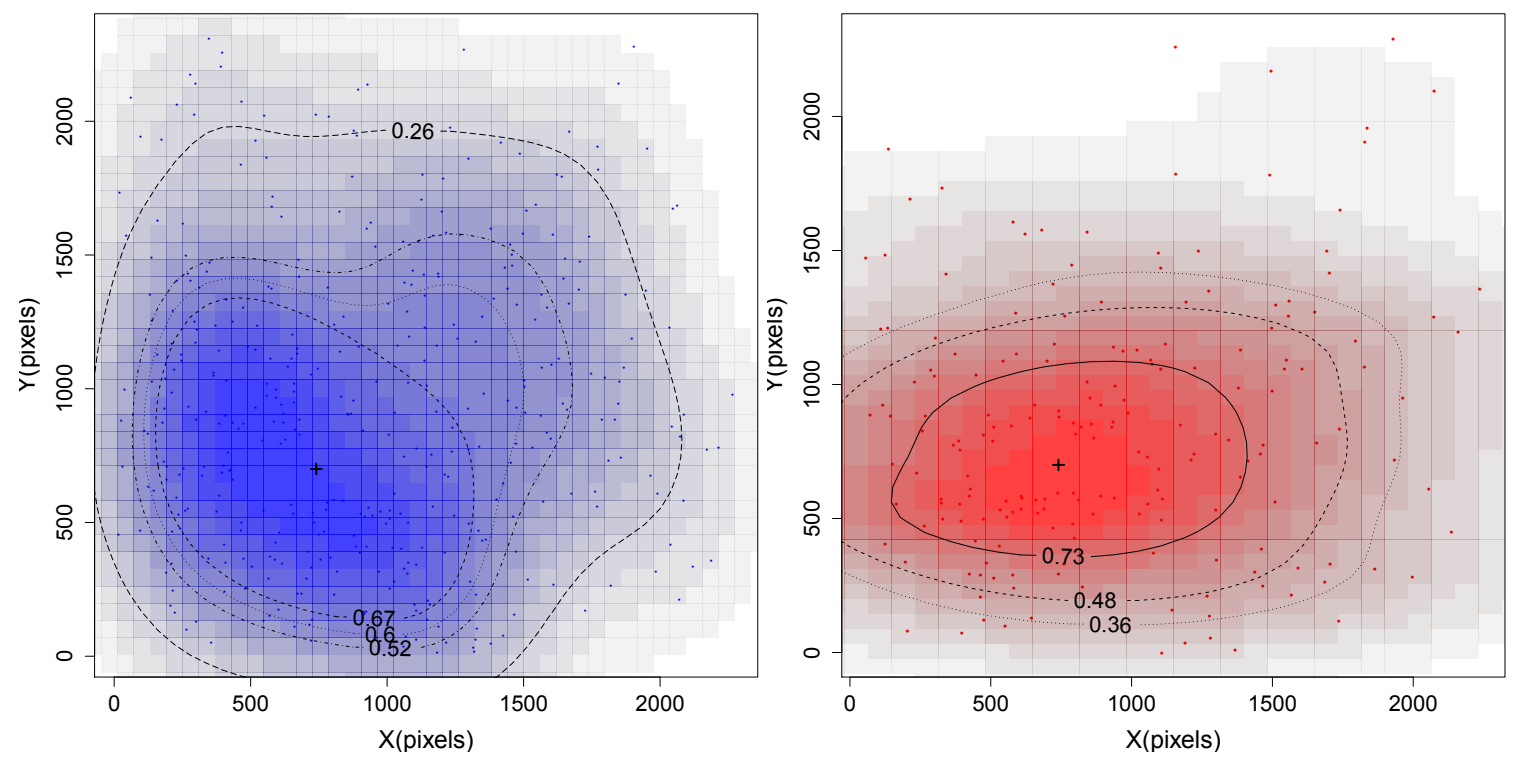

Figure 10. Projected spatial distribution (dots and smoothed distribution) for the blue (left panel) and red (right panel) GC subpopulations. The lines show contours of equal projected number density (darker colours represent higher densities and vice versa). The numbers indicate the value with respect to the maximum. The galaxy centre is marked with a cross.
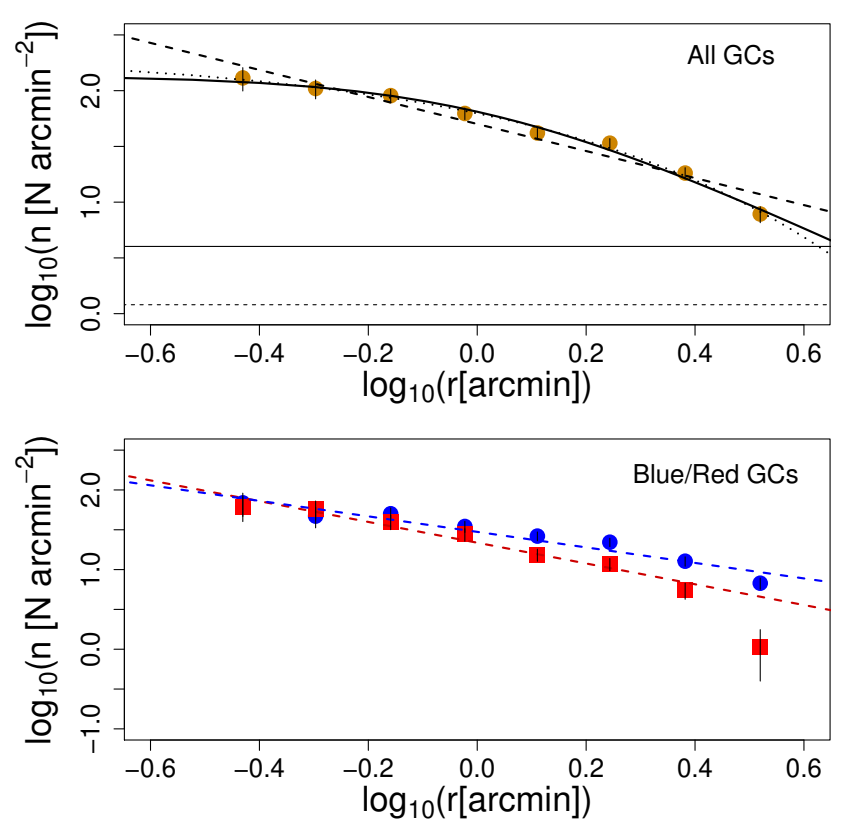

Figure 11. Projected radial distribution, corrected by background and completeness. Upper panel: for the total GC population, lower panel: for the blue and red GC subpopulations (circles and squares, respectively).Solid, dotted, and dashed lines show fits using a Hubble profile, a SÃl'rsic model, and a power-law, respectively.

The solid horizontal line represents the value of the background level and the dotted horizontal line a 30 percent of the background level, which is used to estimate the extension of the GCS.

sinusoidal function:

$N_{\text {red }}=A+B \sin (2 P A+\phi)$

where $N_{\text {red }}$ is the density of red clusters, PA is the posi-
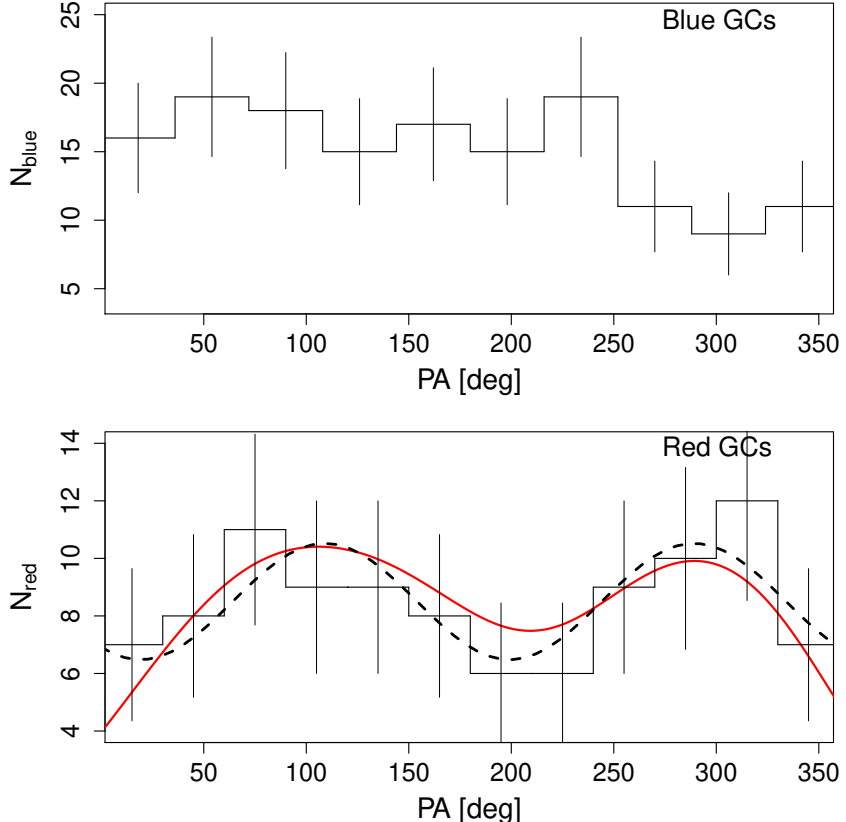

Figure 12. Projected azimuthal distribution for the blue (upper panel) and red (lower panel) GC subpopulations. The dashed and solid lines show the fit of a sinusiodal function (see text) and the smoothed histogram respectively.

tion angle, A is the offset of the symmetry axis, B is the amplitude and $\phi / 2$ is the phase shift.

The parameters resulting from the fit are $A=8.50 \pm 0.36$, $B=-2.02 \pm 0.51$ and $\phi=52^{\circ} \pm 14^{\circ}$. According to them, the $\mathrm{PA}$ of the maximum. i.e. the first over-density, is $\sim 109^{\circ}$.

We also calculated the ellipticity of the projected distribution of the red GCs by means of the expression proposed by Dirsch et al. (2003) and obtained a value of $\epsilon=0.37$. 


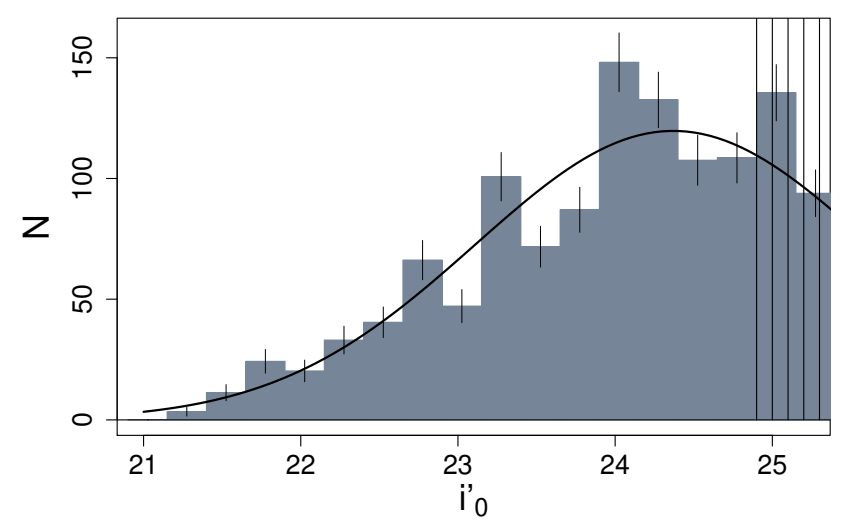

Figure 13. Luminosity function for GC candidates in i'-band, background and completeness-corrected. Errors are estimated as Poisson uncertainties. Solid line shows a Gaussian fit. Vertical lines show the magnitude range that was not considered for the fits.

We note that this analysis of the azimuthal distribution applies to just a fraction of GC candidates, those located within the annulus defined above, while the rest of the GC population is not included. In addition, as the photometry of objects that are close to the borders of the image is usually not very accurate, the outer radius of the annulus was reduced.

\subsection{Luminosity function and GC population}

Fig. 13 shows the background and completeness corrected globular cluster luminosity function (GCLF), using a binwidth of $0.25 \mathrm{mag}$. Two Gaussians were fitted to the GC candidates with i' $\leq 24.9$, excluding fainter ones due to declining completeness. One fit was performed leaving all the parameters free (solid line) and the other one using a fixed mean (turn-over), which was calculated with the adopted distance modulus and an universal absolute visual magnitude $M_{\mathrm{V}_{0}}=-7.4$, taken from Richtler (2003). Afterwards, we converted $V_{0}$ to $i_{0}^{\prime}$ using the transformations given by Bassino \& Caso (2017). There are no notable differences between the two options. Therefore, from here on we will consider the results of the Gaussian fitted with all parameters free. We obtained a turn-over $i_{0}^{\prime}=24.37 \pm 0.25$ with a dispersion of $1.26 \pm 0.20$, that corresponds to a distance modulus $(m-M)=32.37 \pm 0.2$. This value is in agreement within uncertainties with the distance modulus $(m-M)=32.39 \pm 0.14$ given by Tully et al. (2013), which is based on surface brightness fluctuations.

In order to calculate the GC population, we integrated the Hubble law fitted to the radial distribution, assuming that a background-corrected density of 30 per cent of the background sets the limit of the system (see Section 4.4). Afterwards, we applied another correction to take into account that, according to the GCLF, this first result corresponds to only GCs brighter than $i_{0}^{\prime}=24.9$ and we want to consider the whole population. Finally, we obtained a total GC population of $N_{\text {tot }}=2075 \pm 130$ members.

The specific frequency $S_{N}$ is defined as the number of GCs per unit $M_{\mathrm{V}}$ of host galaxy luminosity (Harris \& van

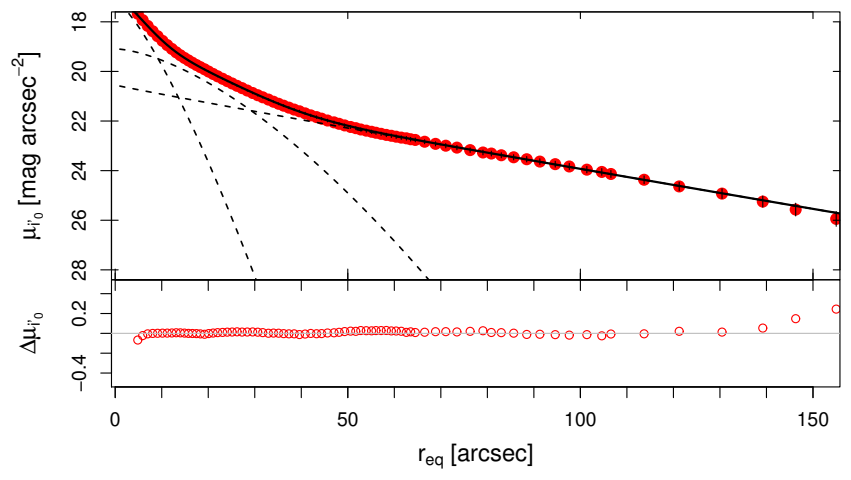

Figure 14. Surface-brightness profile of NGC 3613 in the i'-band (top panel) and resulting residuals (bottom panel). Dashed and solid lines show the three Sérsic models and their sum, respectively. The bottom panel represents the fit residuals.

den Bergh 1981), which was considered to be closely linked to the formation efficiency of GCs (McLaughlin 1999). We obtained a value $S_{N}=5.2 \pm 0.7$, after calculating the absolute $\mathrm{V}$ magnitude $\left(M_{\mathrm{V}}=-21.5 \pm 0.14\right)$ by means of the total $V_{0}$ magnitude obtained from NED and the adopted distance modulus. We can see that the specific frequency of the GCS of NGC 3613 falls within the typical range expected for early-type galaxies with similar luminosity (Brodie \& Strader 2006; Peng et al. 2008; Georgiev et al. 2010; Harris et al. 2013). According to the model of GC formation presented by Kruijssen (2015), where they use the definition of specific frequency normalized by host-galaxy stellar mass, the way GCs form from the interstellar medium in discs and the subsequent disruption they suffer are the main physical processes shaping the behaviour of the specific frequency with respect to galaxy stellar mass.

\section{SURFACE PHOTOMETRY OF NGC 3613}

Fig. 14 (top panel) shows the surface-brightness profile of NGC 3613 in the i'-band (reddening-corrected surface brightness $\mu_{i 0}$ versus equivalent radius $r_{e q}$ ), obtained with IRAF through the ELLIPSE task. We used Sérsic models to fit the galaxy profile and the best fit was provided by the addition of three components, as all fits with less components led to systematic residuals. The expression for each Sérsic model is:

$\mu\left(r_{e q}\right)=\mu_{0}+1.0857\left(\frac{r_{e q}}{r_{0}}\right)^{1 / n}$,

where $\mu$ is the surface-brightness (in units of mag $\left.\operatorname{arcsec}^{-2}\right), \mu_{0}$ is the central surface-brightness, $r_{0}$ is a scale parameter and $n$ is the Sérsic shape index (where $\mathrm{n}=1$ corresponds to an exponential profile and $n=4$ to a de Vaucouleurs profile). The resulting residuals are shown in Fig. 14 (bottom panel). The parameters for the three fitted components are presented in Table 4 . We have also included the respective effective radii, according to the relation $r_{\text {eff }}=b_{n} r_{0}$, where $b_{n}$ is a function of the $\mathrm{n}$ index, that may be estimated with the expression given by Ciotti (1991).

The fitting parameters of our intermediate and outer 


\begin{tabular}{cccc}
\hline Parameter/Component & Inner & Intermediate & Outer \\
\hline \hline$\mu_{0}\left[\right.$ mag arcsec $\left.^{-2}\right]$ & $17.2 \pm 0.1$ & $19.1 \pm 0.1$ & $20.6 \pm 0.1$ \\
\hline$r_{0}[\text { arcsec }]_{n}$ & $5.2 \pm 0.2$ & $17.2 \pm 0.6$ & $30.8 \pm 1.9$ \\
\hline$n$ & $1.32 \pm 0.04$ & $1.58 \pm 0.04$ & $0.96 \pm 0.03$ \\
\hline$r_{\text {eff }}[\mathrm{kpc}]$ & 0.9 & 2.4 & 8.0 \\
\hline
\end{tabular}

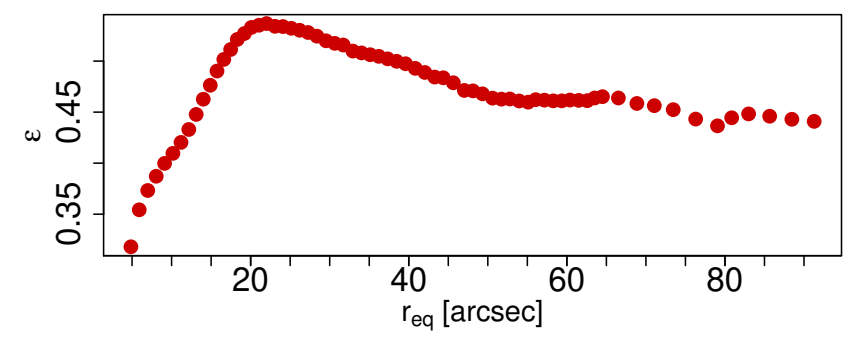

Table 4. Parameters of the three Sérsic components fitted to the observed galaxy profile, i'-band.

components are in agreement within uncertainties with those of the bulge and exponential disk obtained by Krajnović et al. (2013) (ATLAS ${ }^{3 D}$ project) through a two-component fit. In particular, they point out that the median SÃl'rsic index of the bulge is $n=1.7$ for galaxies classified as fast rotators, i.e. close to our value for NGC $3613(n=1.6)$.

The presence of three components in massive E galaxies, like our present target, has already been pointed out by several authors. For instance, Huang et al. (2013a) present a study of nearby Es from the Carnegie-Irvine Galaxy Survey and show that two-dimensional surface-brightness distributions of most of them, can be described by a compact core as inner component, an intermediate component as main body, and an outer envelope. For a sample close to 100 galaxies, they obtain Sérsic index $n \approx 1-2$ for the components, in agreement with the values obtained for NGC 3613 though we perform a one-dimensional analysis. Multi-components in this type of galaxies (Huang et al. 2013a,b; Oh et al. 2017 , and references therein) are understood as the consequence of a two-phase formation scenario. At high redshift $(\mathrm{z} \geq 3)$, the evolution is dominated by in-situ star formation owing to highly dissipative processes, from which the inner substructure of the galaxies derive. On the other side, the outer extended envelopes were built-up during a later phase, mainly dominated by accretion through 'dry' minor mergers.

Fig. 15 shows the parameters of the isophotes obtained with ELLIPSE for NGC 3613, as a function of $r_{e q}$. That is, ellipticity $\epsilon$ (top panel), position angle PA measured positive from $\mathrm{N}$ to $\mathrm{E}$ (middle panel), and $\mathrm{A} 4$ Fourier coefficient which represents disky and boxy isophotes for $A 4>0$ and $A 4<0$, respectively (bottom panel). The values of $\epsilon$ are mostly higher than 0.4, which is typical of fast rotators as NGC 3613 (Cappellari et al. 2011b). Changes in the isophotal parameters, at $r_{e q} \sim 20$ and $\sim 55$ arcsec, agree with the dominance of different components in the brightness profile. Fig. 16 shows the final combined GMOS image (i'-band) of NGC 3613, where the boxy shape of the outer isophotes is evident $(A 4<0)$. Five UCD candidates have been identified with squares in the surroundings. Globally, $\epsilon$ and PA agree with those given by (Krajnović et al. 2011) in the context of the $\operatorname{ATLAS}^{3 D}$ project.

Fig. 17 shows the GMOS image obtained by subtracting, from the original image, a smoothed model of the surfacebrightness distribution of the galaxy, performed with ELLIPSE and BMODEL. In this residual image, there is an observable substructure at a low surface-brightness level. There is a plume towards the left side of the galaxy, pointing to the south, that is detectable in the original image (Fig. 16)

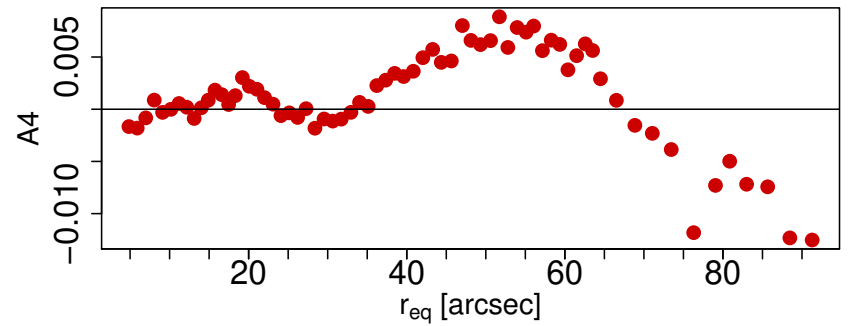

Figure 15. Parameters of the isophotes fitted with ELLIPSE to the NGC 3613 surface-brightness distribution, as a function of $r_{e q}$. From top to bottom panels: ellipticity $\epsilon$, position angle PA, and $A 4$ Fourier coefficient, respectively. The horizontal line in the bottom panel corresponds to $A 4=0$.

so that it cannot be a spurious residual of the image processing. Another plume is present on the opposite side, pointing to the north too. A bright $\mathrm{x}$-shape residual located in the central region may be connected to these plumes. An inner stellar disk is aligned with the major axis of the galaxy isophotes (see also Ebneter et al. 1988). All this lying substructure can be understood as another indication of the multi-components identified in the galaxy, related to the formation history, where the plumes may be tidal remainings of past accretions (e.g. Barnes \& Hernquist 1992; Hernquist \& Spergel 1992). On the other hand, we find no clear evidence of interaction with NGC 3610.

\section{DISCUSSION}

\subsection{Relation between GC subpopulations and the host galaxy}

In many early-type galaxies, a close relationship has been observed between its stellar component and the red GC subpopulation, detected in the kinematics (e.g. Pota et al. 2013), in their radial projected distributions (e.g. Ko et al. 2019 ) as well as the shape (measured by $\epsilon$ ) of the red clusters and the stellar light distribution (e.g. Park \& Lee 2013). In 


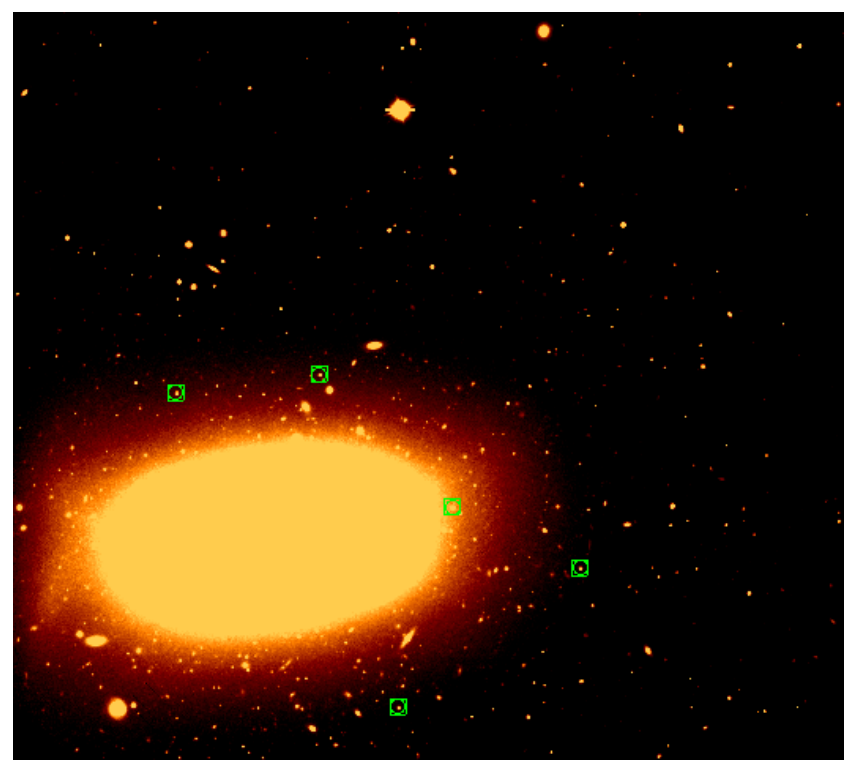

Figure 16. GMOS final image containing NGC 3613 (i'-band). UCD candidates are highlighted with green squares. North is up, east to the left.

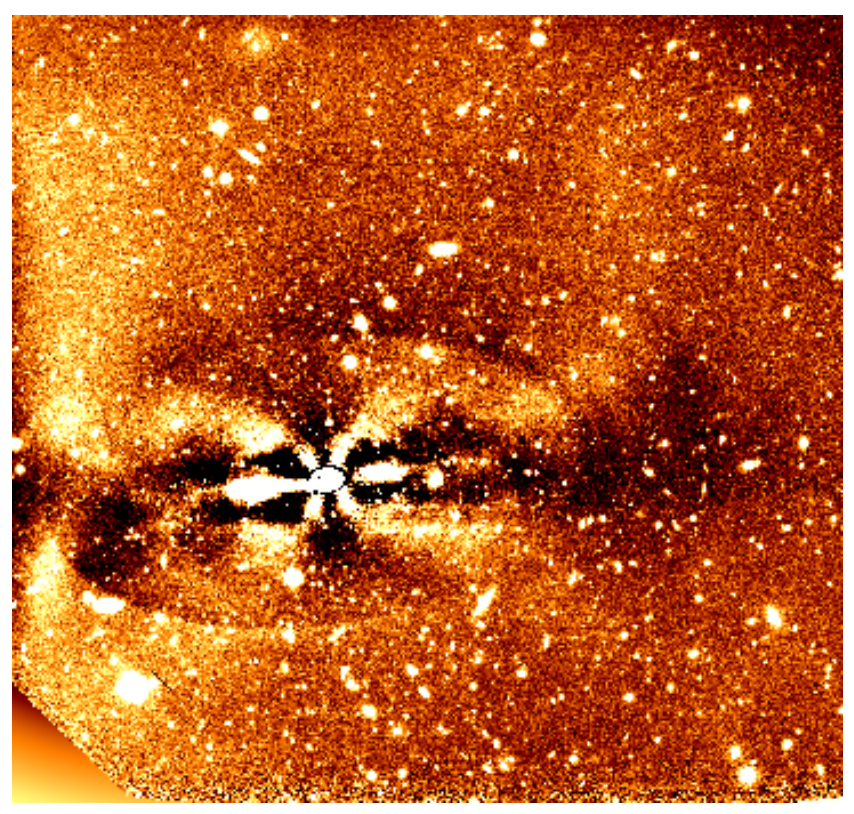

Figure 17. Residual GMOS image of NGC 3613 (i'-band, FOV: $4.3 \times 4.3 \operatorname{arcmin}$, scale $=0.146 \operatorname{arcsec}$ pixel $^{-1}$ )

, obtained by subtracting a smooth model of the galaxy light. Plumes in the outskirts and inner substructure are discernible. North is up, east to the left.

the case of NGC 3613, we do not have enough radial coverage to determine colour gradients but can analyse the trend of mean colours for blue and red GCs, at three different radial ranges (Table 2). The blue peak of the inner radial range is redder than those of the intermediate and outer ranges, while the red peak does not present any clear variation with radius. That the blue peak gets bluer with increasing radius is in agreement with massive elliptical galaxies located at the centre of clusters (e.g., Bassino et al. 2006 in Fornax, Caso et al. 2017 in Antlia), although NGC 3613 is considered as just the central galaxy of a group.

Regarding the red GC population, we noticed that the PA of the two over-densities detected in their projected azimuthal distribution (i.e., $\mathrm{PA} \sim 110^{\circ}$ and $\sim 290^{\circ}$ ) correspond, as expected, to the orientation of the major-axis of the galaxy elliptical contours. In addition, the ellipticity of the projected distribution of the red clusters resulted $\epsilon=0.37$. We calculated a mean $\epsilon$ and PA for the host galaxy isophotes with semi-axis between $48<R_{g}<102$ (i.e. the same radial range used for the GC azimuthal distribution), resulting $<\epsilon>=0.47(\sigma=0.017)$ and $\langle P A\rangle=97^{\circ}(\sigma=0.14)$. The shape parameters of the light distribution are very similar to those of the projected red GC distribution, while there is no obvious relation to the blue GCs.

Both effects can be related to the formation history of the galaxy as it is generally accepted that most massive early-type galaxies in the local Universe form in two-phases (Forbes et al. 2011; van Dokkum et al. 2015).

\subsection{Ultra-compact dwarf candidates}

Five UCD candidates have been detected in the colourmagnitude diagram of point-sources, shown in Fig. 5 with empty circles. This small sample, according to our photometry, shows colours within the range corresponding to GCs but their $i^{\prime}$ magnitudes are brighter than expected for a GC (assuming a limit at $i_{0}^{\prime}=20.8$, as explained in Section 4.1).

The positions of these UCD candidates are also identified on our GMOS image (Fig. 16), where they appear surrounding NGC 3613 at galactocentric radius between 66 and 121 arcsec, i.e. well within the radial range covered by the GC candidates. Their colours are in the range $0.85<(g-$ $i)_{0}<1.02$ and their absolute magnitudes $-11.8<M_{i}<-11.5$ according to adopted distance. If we compare to the $M_{i}$ versus $(g-i)_{0}$ colour-magnitude diagram presented by Brodie et al. (2011, their fig. 5) for the sample of M 87 UCDs, our candidates fall in the same locus as the M 87 ones.

We plan to obtain spectra of these UCD candidates in the near future, in order to confirm membership with radial velocities and analyse physical properties like metallicity, age, stellar populations, etc.

\section{SUMMARY AND CONCLUSIONS}

We present the first photometric study of the GCS of the bright elliptical galaxy NGC 3613, that is located at the centre of a galaxy group but has an intrinsic brightness typical of a brightest cluster galaxy. On the basis of $g^{\prime}, r^{\prime}, i^{\prime}$ Gemini/GMOS images, not only the properties of the GCS but also the surface-photometry of the host galaxy were investigated. In addition, its distance was confirmed by means of the GC luminosity function and five new UCD candidates were discovered. The principal results are summarised here:

- The GC colour distribution is bimodal, considering the whole sample or three different radial ranges. The mean colour of the blue GCs gets slightly bluer for increasing radius, which is understood as a hint that these metal-poor clusters may have been accreted with satellite galaxies. 
- Regarding the blue GC subpopulation, they follow a colour-magnitude relation in the sense that brighter clusters get redder, i.e. the so-called blue tilt, for whose interpretation several scenarios have been proposed. No equivalent relation is present in the red GC subpopulation.

- Regarding the red GC subpopulation, its spatial, radial and azimuthal projected distributions show that they are more concentrated towards the host galaxy and trace closely the shape of the galaxy light isophotes. Thus, these effects point to a common origin of the galaxy stellar component and the majority of metal-rich GCs. The blue GC subpopulation presents a mostly uniform and more extended projected distribution.

- By means of the turn-over of the GC luminosity function, we obtain a distance of $29.8 \pm 2.8 \mathrm{Mpc}$, in agreement within uncertainties with the initially adopted value of $30.1 \mathrm{Mpc}$ (Tully et al. 2013). The total GC population is estimated in $N_{\text {tot }}=2075 \pm 133$ GCs and the specific frequency $S_{N}=5.2 \pm 0.7$. Both values are typical for GCSs in host galaxies of similar luminosity than NGC 3613 .

- There is a noticeable substructure in the surfacebrightness distribution of NGC 3613, detected in the original and residual images. It may be a sign of past tidal interactions but cannot be clearly related to any interplay with its neighbour, the merger remnant NGC 3610. We also find no evidence of such interaction in the GC projected distributions.

- We find a sample of five new UCD candidates in the outskirts of NGC 3613, brighter than the regular GCs but within the same colour range. We plan to continue studying them with spectroscopy in the near future.

\section{ACKNOWLEDGEMENTS}

We thank the constructive comments of the referee, which helped to improve this paper. This work was funded with grants from Consejo Nacional de Investigaciones Científicas y Técnicas de la República Argentina, Agencia Nacional de Promoción Científica y Tecnológica, and Universidad Nacional de La Plata, Argentina.

Based on observations obtained at the Gemini Observatory (programme GN2013A-Q-42, PI: J.P. Caso), which is operated by the Association of Universities for Research in Astronomy, Inc., under a cooperative agreement with the NSF on behalf of the Gemini partnership: the National Science Foundation (United States), the National Research Council (Canada), CONICYT (Chile), the Australian Research Council (Australia), Ministério da Ciência, Tecnologia e Inovação (Brazil) and Ministerio de Ciencia, Tecnología e Innovación Productiva (Argentina). This research has made use of the NASA/IPAC Extragalactic Database (NED) which is operated by the Jet Propulsion Laboratory, California Institute of Technology, under contract with the National Aeronautics and Space Administration.

\section{REFERENCES}

Alamo-Martínez K. A., et al., 2013, ApJ, 775, 20

Amorisco N. C., 2019, MNRAS, 482, 2978

Barnes J. E., Hernquist L., 1992, ARA\&A, 30, 705

Bassino L. P., Caso J. P., 2017, MNRAS, 466, 4259
Bassino L. P., Faifer F. R., Forte J. C., Dirsch B., Richtler T., Geisler D., Schuberth Y., 2006, A\&A, 451, 789

Bassino L. P., Richtler T., Dirsch B., 2008, MNRAS, 386, 1145

Bertin E., Arnouts S., 1996, A\&AS, 117, 393

Binney J., Tremaine S., 1987, Galactic dynamics

Brodie J. P., Strader J., 2006, ARA\&A, 44, 193

Brodie J. P., Romanowsky A. J., Strader J., Forbes D. A., 2011, AJ, 142, 199

Cappellari M., et al., 2011a, MNRAS, 413, 813

Cappellari M., et al., 2011b, MNRAS, 416, 1680

Caso J. P., Richtler T., Bassino L. P., Salinas R., Lane R. R., Romanowsky A., 2013, A\&A, 555, A56

Caso J. P., Bassino L. P., Gómez M., 2015, MNRAS, 453, 4421

Caso J. P., Bassino L. P., Gómez M., 2017, MNRAS, 470, 3227

Caso J. P., Bassino L. P., Richtler T., Salinas R., 2019, MNRAS, 483,4371

Chies-Santos A. L., Larsen S. S., Cantiello M., Strader J., Kuntschner H., Wehner E. M., Brodie J. P., 2012, A\&A, 539, A54

Ciotti L., 1991, A\&A, 249, 99

Dirsch B., Richtler T., Bassino L. P., 2003, A\&A, 408, 929

Durrell P. R., et al., 2014, ApJ, 794, 103

Ebneter K., Djorgovski S., Davis M., 1988, AJ, 95, 422

Emsellem E., et al., 2011, MNRAS, 414, 888

Escudero C. G., Faifer F. R., Bassino L. P., Calderón J. P., Caso J. P., 2015, MNRAS, 449, 612

Escudero C. G., Faifer F. R., Smith Castelli A. V., Forte J. C., Sesto L. r. A., González N. M., Scalia M. C., 2018, MNRAS, 474, 4302

Faifer F. R., et al., 2011, MNRAS, 416, 155

Ferrarese L., et al., 2012, ApJS, 200, 4

Forbes D. A., Spitler L. R., Strader J., Romanowsky A. J., Brodie J. P., Foster C., 2011, MNRAS, 413, 2943

Forbes D. A., Ponman T., O'Sullivan E., 2012, MNRAS, 425, 66

Forte J. C., Faifer F. R., Vega E. I., Bassino L. P., Smith Castelli A. V., Cellone S. A., Geisler D., 2013, MNRAS, 431, 1405

Georgiev I. Y., Puzia T. H., Goudfrooij P., Hilker M., 2010, MNRAS, 406, 1967

Hansen B. M. S., et al., 2013, Nature, 500, 51

Harris W. E., 2009, ApJ, 703, 939

Harris W. E., van den Bergh S., 1981, AJ, 86, 1627

Harris W. E., Whitmore B. C., Karakla D., Okoń W., Baum W. A., Hanes D. A., Kavelaars J. J., 2006, ApJ, 636, 90

Harris W. E., Harris G. L. H., Alessi M., 2013, ApJ, 772, 82

Harris W. E., et al., 2014, ApJ, 797, 128

Harris W. E., Blakeslee J. P., Whitmore B. C., Gnedin O. Y., Geisler D., Rothberg B., 2016, ApJ, 817, 58

Hernquist L., Spergel D. N., 1992, ApJ, 399, L117

Huang S., Ho L. C., Peng C. Y., Li Z.-Y., Barth A. J., 2013a, ApJ, 766, 47

Huang S., Ho L. C., Peng C. Y., Li Z.-Y., Barth A. J., 2013b, ApJ, 768, L28

Jordán A., et al., 2007, ApJS, 169, 213

Kartha S. S., Forbes D. A., Spitler L. R., Romanowsky A. J., Arnold J. A., Brodie J. P., 2014, MNRAS, 437, 273

Ko Y., Lee M. G., Park H. S., Lim S., Sohn J., Hwang N., Park B.-G., 2019, ApJ, 872, 202

Krajnović D., et al., 2011, MNRAS, 414, 2923

Krajnović D., et al., 2013, MNRAS, 432, 1768

Kruijssen J. M. D., 2015, MNRAS, 454, 1658

Kruijssen J. M. D., Pelupessy F. I., Lamers H. J. G. L. M., Portegies Zwart S. F., Bastian N., Icke V., 2012, MNRAS, 421, 1927

Kruijssen J. M. D., Pfeffer J. L., Crain R. A., Bastian N., 2019, MNRAS, 486, 3134

Madore B. F., Freedman W. L., Bothun G. D., 2004, ApJ, 607, 810

McDermid R. M., et al., 2015, MNRAS, 448, 3484

McLaughlin D. E., 1999, AJ, 117, 2398 
Mieske S., Hilker M., Infante L., Jordán A., 2006, AJ, 131, 2442

Muratov A. L., Gnedin O. Y., 2010, ApJ, 718, 1266

Norris M. A., et al., 2008, MNRAS, 385, 40

Norris M. A., et al., 2012, MNRAS, 421, 1485

O'Sullivan E., et al., 2017, MNRAS, 472, 1482

Oh S., Greene J. E., Lackner C. N., 2017, ApJ, 836, 115

Oldham L. J., Auger M. W., 2016, MNRAS, 455, 820

Park H. S., Lee M. G., 2013, ApJ, 773, L27

Peng E. W., et al., 2008, ApJ, 681, 197

Pfeffer J., Kruijssen J. M. D., Crain R. A., Bastian N., 2018, MNRAS, 475, 4309

Pota V., et al., 2013, MNRAS, 428, 389

Powalka M., et al., 2016, ApJ, 829, L5

Richtler T., 2003, in Alloin D., Gieren W., eds, Lecture Notes in Physics, Berlin Springer Verlag Vol. 635, Stellar Candles for the Extragalactic Distance Scale. pp 281-305 (arXiv:astroph/0304318), doi:10.1007/978-3-540-39882-0_15

Schlafly E. F., Finkbeiner D. P., 2011, ApJ, 737, 103

Schuberth Y., Richtler T., Hilker M., Dirsch B., Bassino L. P., Romanowsky A. J., Infante L., 2010, A\&A, 513, A52

Schweizer F., Seitzer P., 1992, AJ, 104, 1039

Sersic J. L., 1968, Atlas de Galaxias Australes

Sesto L. A., Faifer F. R., Forte J. C., 2016, MNRAS, 461, 4260

Smith J. A., et al., 2002, AJ, 123, 2121

Tonini C., 2013, ApJ, 762, 39

Tully R. B., et al., 2013, AJ, 146, 86

Usher C., et al., 2012, MNRAS, 426, 1475

Usher C., Forbes D. A., Spitler L. R., Brodie J. P., Romanowsky A. J., Strader J., Woodley K. A., 2013, MNRAS, 436, 1172

Wehner E. M. H., Harris W. E., Whitmore B. C., Rothberg B., Woodley K. A., 2008, ApJ, 681, 1233

de Vaucouleurs G., de Vaucouleurs A., Corwin Jr. H. G., Buta R. J., Paturel G., Fouqué P., 1991, Third Reference Catalogue of Bright Galaxies. Volume I: Explanations and references. Volume II: Data for galaxies between $0^{h}$ and $12^{h}$. Volume III: Data for galaxies between $12^{h}$ and $24^{h}$.

van Dokkum P. G., et al., 2015, ApJ, 813, 23

This paper has been typeset from a $\mathrm{T}_{\mathrm{E}} \mathrm{X} / \mathrm{LAT}_{\mathrm{E} X} \mathrm{X}$ file prepared by the author. 